\title{
How Healthcare Professionals Comprehend Process Models - An Empirical Eye Tracking Analysis
}

\author{
Michael Winter ${ }^{1}$, Cynthia Bredemeyer ${ }^{2}$, Manfred Reichert ${ }^{1}$, Heiko Neumann ${ }^{3}$, Thomas Probst ${ }^{4}$, Rüdiger Pryss ${ }^{2}$ \\ ${ }^{1}$ Institute of Databases and Information Systems, Ulm University, Germany \\ ${ }^{2}$ Institute of Clinical Epidemiology and Biometry, University of Würzburg, Germany \\ ${ }^{3}$ Institute of Neural Information Processing, Ulm University, Germany \\ ${ }^{4}$ Department for Psychotherapy and Biopsychosocial Health, Danube University Krems, Austria \\ \{michael.winter, manfred.reichert, heiko.neumann\}@uni-ulm.de, ruediger.pryss@uni-wuerzburg.de, \\ cynthia.bredemeyer@stud-mail.uni-wuerzburg.de, thomas.probst@ donau-uni.ac.at
}

\begin{abstract}
Digitization is advancing rapidly in many prevalently analogue domains such as healthcare. For the latter domain, the synergies with modern information technologies (IT) have become an integral part regarding communication and collaboration. For this reason, a comprehensible language is of importance in order to allow a frictionless exchange of information between domain experts. The Business Process Model and Notation (BPMN) 2.0 represents a promising notation that may be applied as lingua franca. Although the BPMN 2.0 is widespread applied by experts in business and industry, little experience exists how BPMN 2.0 is adopted in healthcare. In order to assess how BPMN 2.0 is deployed in healthcare, we conducted a preliminary eye tracking study, in which $n=16$ professionals from healthcare comprehended a particular BPMN 2.0 process model. The results indicate that BPMN 2.0 might be a candidate for a lingua franca to foster the comprehensible exchange of information as well as collaboration between healthcare and IT.

Index Terms-Business Process Model and Notation 2.0, Process Model Comprehension, Study, Eye Tracking, Healthcare
\end{abstract}

\section{INTRODUCTION}

Technological advancements in the context of digitization are rapid and they are changing the way we work, communicate, and collaborate. Digitization no longer solely affects the traditional information technology (IT) domain, but domains across all industries such as economy, education, and healthcare [1]. Regarding the latter, the ever-increasing linkage of IT with the analogue healthcare world leads to new opportunities (e.g., patients receive optimized treatment plans [2] or operations can be performed remotely [3]). However, not only opportunities emerge, but new challenges arise that have to be thoroughly addressed [4]. In order to cope with these challenges in healthcare, it is of utmost importance to provide a language, which allows for the comprehensible exchange of information between the IT and healthcare professionals (i.e., lingua franca). A best practice in this context is the use of visual process models [5], which conceptualize, determine, and describe procedures, technical systems, or the processes of organizations for the purpose of proper documentation, instant communication, effective analysis, and collaboration [6].

In order to benefit from the merits of process models, a proper comprehension of such models must be ensured [7]. Therefore, it is important to understand how individuals read and comprehend process models, including positive and negative factors that affect model comprehension. If factors that incapacitate process model comprehension are not addressed properly, respective processes might not deliver the required results. Prior research demonstrated that not only process model expertise is a decisive factor, but process model comprehension also depends on other person-related characteristics [8]. In this context, the utilization of eye tracking has become a valuable instrument to investigate comprehension strategies, difficulties to comprehend model constructs, or the cognitive load during the comprehension of such models [9].

This paper presents the results from an exploratory eye tracking study about how healthcare professionals read and comprehend process models. Participants from healthcare needed to comprehend a BPMN 2.0 process model, for which we relied on eye tracking to record eye movements during model comprehension. An emphasis was put on person-related characteristics (e.g., gender, age) and their influence on the comprehension of such models. The results indicate that the presented process model could be comprehended intuitively, but that there were differences in comprehension based on several characteristics. The insights provide further information towards a lingua franca to improve communication and collaboration between IT and healthcare professionals.

Related work is presented in Section II. The study setting is described in Section III, while Section IV discusses the obtained results. Finally, Section V concludes the paper.

\section{RELATED WORK}

Since process models are becoming increasingly relevant in digitization, a vast body of research has evolved in the context of process model comprehension over the last decade. Research studied objective as well as subjective comprehensibility and the inherent factors with their influence on the comprehension of such models. Regarding objective comprehensibility, structural properties of a process models have been considered [7], [10]. In the context of subjective comprehensibility, process model reader related factors such as model expertise [11] or model reader preferences [12] were investigated. As known from other domains, emphasis is increasingly put on cognitive aspects. For example, cognitive style [13] 
TABLE I

DEMOGRAPHIC DATA

\begin{tabular}{|c|c|c|c|c|c|c|c|c|c|c|c|c|c|}
\hline ID & Gender & Age & Prof. & Exp. & Tech. Prof. & Tech. Lei. & ID & Gender & Age & Prof. & Exp. & Tech. Prof & Tech. Lei. \\
\hline$\overline{1} 1$ & female & $26-35$ & Epi. & $\overline{\mathrm{No}}$ & No & No & 9 & female & $26-35$ & Eco. & $\overline{\text { Yes }}$ & No & No \\
\hline 2 & male & $26-35$ & Eco. & Yes & No & No & 10 & female & $20-25$ & Eco. & Yes & No & No \\
\hline 3 & male & $20-25$ & Eco. & No & No & No & 11 & male & $26-35$ & Com. & Yes & Yes & Yes \\
\hline 4 & male & $26-35$ & Com. & Yes & Yes & Yes & 12 & female & $20-25$ & Bio. & No & Yes & No \\
\hline 5 & female & $20-25$ & Com. & Yes & Yes & No & 13 & male & $26-35$ & Com. & Yes & Yes & Yes \\
\hline 6 & male & $26-35$ & Med. & No & Yes & No & 14 & male & $26-35$ & Med. & Yes & Yes & No \\
\hline 7 & female & $26-35$ & Med. & No & No & No & 15 & male & $26-35$ & Med. & No & No & No \\
\hline 8 & female & $20-25$ & Bio. & No & Yes & No & 16 & female & $20-25$ & Psy. & No & No & No \\
\hline
\end{tabular}

or cognitive bias [14]. Furthermore, many technology-driven approaches like the use of electrodermal activity [15] are performed for a more fine-grained investigation of cognitive aspects and their influence during process model comprehension. Especially the application of eye tracking is becoming prominent for research on process model comprehension (e.g., [16]-[18]). Finally, the isolated consideration of specified collectives during process model comprehension has revealed various new insights [19]. However, to understand how healthcare professionals read and comprehend process models to bridge the gap between IT and healthcare still needs more investigations like shown in this paper.

\section{Study SetTing}

Healthcare professionals face a plethora of challenges when performing their processes everyday [20]. Through the digitization, it is becoming increasingly apparent that processes, communication, and medical applications in healthcare are inadequately supported by the IT [21]. Therefore, it is important to foster a lingua franca in order to effectively facilitate the adoption of digitization and to benefit from the associated advantages. One promising approach constitutes the application of process models, which can be utilized for the communication of information as well as collaboration. For an efficient use of process models, it must be ensured that they are comprehended correctly. Prior research showed that process models can be comprehended intuitively, but especially those without any process model expertise show difficulties in coping with the complexities involved in parsing the relevant information in such models [8]. Since IT and healthcare coalesce in digitization, it is mandatory to investigate how healthcare professionals read and comprehend process models. Therefore, this study was carried out as part of a healthcare research project by a student of translational medicine (with dental background). The study had the goal to assess the comprehension of a process model by healthcare professionals to evaluate the suitability of BPMN 2.0 as lingua franca for the communication between IT and healthcare professionals.

\section{A. Study Planning}

Participants: The study included $\mathrm{n}=16$ participants from different disciplines in healthcare (i.e., 1 epidemiologist, 4 economists, 4 computer scientists, 4 medical practitioners, 2 biologists, and 1 psychologist; see Table I). Emphasis was put on the following person-related characteristics and their influence on the comprehension of process models: gender, age, profession, process model expertise, and technology affinity in profession as well as leisure. Based on these person-related characteristics, the participants were divided into different groups, which shared the same characteristics. Note that participants could belong to several groups.

Materials: In this study, a BPMN 2.0 process model was used [22]. The choice to use a BPMN 2.0 process model was made for several rationales: BPMN 2.0 is the de facto industry standard for the creation of comprehensible process models, plus it is an ISO/IEC 1950:2013 norm. During the last decade, a vast body of knowledge evolved, which has promoted the widespread application of BPMN 2.0. The used process model was composed of basic as well as advanced modeling elements and contained a total number of 41 elements (excluding the edges). Thematically, it documented a dental appointment with all relevant participants, functions, and resources (see Fig. 1). Thereby, particular emphasis was put in the comprehension of the modeling constructs decision, loop, and parallelism, as these constructs usually require modeling expertise.

\section{Performance Measures:}

1) Number of fixations: Fixations constitute eye movements to deploy over attention by analyzing content (e.g., images) with high resolution spectral analysis [23]. Further, fixation sequence analyses (i.e., sequential analysis of attentional content reading) allowed us to make conclusion about the cognitive load as well as about specific points in the stimulus that may pose a challenge in the comprehension process.

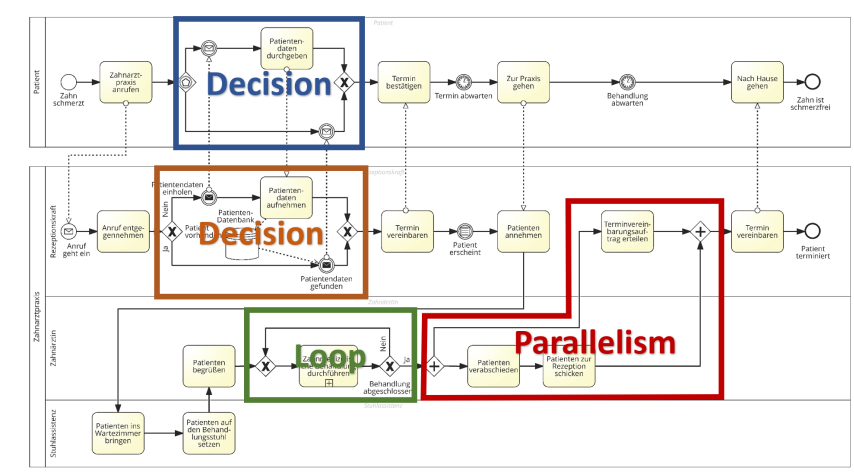

Fig. 1. BPMN 2.0 process model with highlighted modeling constructs decision, loop, and parallelism 
TABLE II

DESCRIPTIVE RESULTS

\begin{tabular}{|c|c|c|c|c|c|c|c|c|c|c|c|c|c|c|c|c|c|}
\hline ID & Fix. & Fix. Dur. & Score & ICL & ECL & GCL & PUU & PEU & ID & Fix. & Fix. Dur. & Score & ICL & ECL & GCL & PUU & PEU \\
\hline 1 & - & - & 7 & 3.5 & 2 & 6 & 17 & 17 & 9 & 605 & 211.57 & 7 & 3.5 & 2 & 5.5 & 16 & 15 \\
\hline 2 & 528 & 226.61 & 7 & 2 & 1 & 7 & 15 & 18 & 10 & 704 & 179.13 & 6 & 3 & 2.6 & 4.5 & 16 & 15 \\
\hline 3 & 594 & 218.34 & 7 & 2 & 3 & 6 & 11 & 16 & 11 & 610 & 264.69 & 7 & 3 & 2 & 6 & 18 & 16 \\
\hline 4 & - & - & 5 & 1.5 & 1.3 & 6 & 12 & 15 & 12 & 757 & 226.01 & 5 & 5 & 7 & 7 & 23 & 15 \\
\hline 5 & 723 & 232.54 & 3 & 3.5 & 1.3 & 7 & 17 & 17 & 13 & 539 & 201.43 & 4 & 3.5 & 3.6 & 7 & 17 & 16 \\
\hline 6 & - & - & 5 & 5.5 & 2.6 & 5.5 & 16 & 16 & 14 & 591 & 311.95 & 7 & 3.5 & 2.3 & 6 & 14 & 14 \\
\hline 7 & 631 & 213.97 & 6 & 4 & 3 & 4.5 & 14 & 13 & 15 & 753 & 163.36 & 6 & 4 & 5.6 & 6 & 12 & 11 \\
\hline 8 & 677 & 185.32 & 7 & 5 & 4.3 & 7 & 16 & 15 & 16 & 767 & 231.32 & 5 & 4 & 3 & 6.5 & 19 & 18 \\
\hline All & $\begin{array}{l}652.53 \\
(79.42)\end{array}$ & $\begin{array}{l}220.46 \\
(36.53)\end{array}$ & $\begin{array}{l}5.87 \\
(1.22)\end{array}$ & $\begin{array}{l}3.53 \\
(1.06)\end{array}$ & $\begin{array}{l}2.93 \\
(1.55)\end{array}$ & $\begin{array}{l}6.10 \\
(0.79)\end{array}$ & $\begin{array}{l}15.43 \\
(1.73)\end{array}$ & $\begin{array}{l}15.81 \\
(2.83)\end{array}$ & & & & & & & & & \\
\hline
\end{tabular}

2) Fixation duration: The fixation duration indicates the period of time in which the eyes remain still while looking at a stimulus [24]. During this time period, the acquisition of information from the viewed point in a stimulus (i.e., process model) takes place. Hence, the analysis of the average fixation duration allowed us for additional assumptions regarding the cognitive load during process model comprehension.

3) Score: Participants needed to answer eight true-or-false comprehension questions about the presented process models. The comprehension questions referred to the semantic and syntactic dimensions of the process model.

4) Cognitive load: The cognitive load depicts the invested cognitive capacity of the working memory during a task and consists of the following dimensions: intrinsic, extraneous, and germane cognitive load [25]. Intrinsic load constitutes the complexity of intrinsic information and is affected by existing knowledge and element interactivity (e.g., demand on the working memory). In turn, extraneous load is affected by the way information is presented. Finally, germane load describes the mental effort to process and comprehend information based on constructed mental models. he adapted measurement proposed in [26] was used for the study to measure the dimensions. The single dimensions, which were comprised of several items (i.e., two for intrinsic, three for extraneous, and germane cognitive load), were assessed on a psychometrically standardized questionnaire (i.e., 7-point Likert scale).

5) Perceived usefulness for understandability (PUU): It describes the perceived usefulness of the process model and is a part of the technology acceptance model (TAM) [27]. Therefore, four items on a 7-point Likert scale from strongly disagree (i.e., 1) to strongly agree (i.e., 7) needed to be answered, resulting in a $\min / \max$ value of $(4 \times 7)$ [28].

6) Perceived ease of understandability (PEU): Derived from TAM, PEU characterizes that the application of process models is associated with less mental effort. Therefore, four items on a 7-point Likert scale from strongly disagree (i.e., 1) to strongly agree (i.e., 7) needed to be answered, resulting in a $\mathrm{min} / \mathrm{max}$ value of $(4 \times 7)$ [28].

\section{B. Study Design and Procedure}

The study was conducted in a designated lab at the University of Würzburg. Due to the coronavirus (COVID-19) pandemic, a dedicated study procedure was carried out in compliance with hygiene regulations. Therefore, only one participant could be evaluated at each study session. A session took about 20 minutes and was as follows: The participant was welcomed and first of all, he or she needed to sign a COVID19 declaration. Afterwards, the study procedure was explained and an informed consent from the participant was obtained. Then, the participant was asked to answer a set of demographic questions for collecting relevant person-related characteristics (e.g., age or gender). Following this, the used eye tracker was calibrated with a 13-point screen-based calibration to ensure a precise recording of the eye movements. After these steps, the dental appointment BPMN 2.0 process model was presented to the participant for exactly 03:30 minutes. During this time, the participant had to read and comprehend the process model, whilst their eye movements were recorded. The presentation time was defined by three process modeling experts in a consensus-building process, which has been deemed sufficient to read and comprehend all relevant information in the process model. After the comprehension task, the participant needed to answer a questionnaire capturing the cognitive load and the level of acceptability. Then, a comprehension task with eight questions regarding the prior comprehended process model was handed over in order to check the comprehensibility. Finally, after the opportunity to leave feedback, the study ended.

Instrumentation: COVID-19 declaration, demographic data, comprehension question score, and information related to the cognitive load as well as the level of acceptability was collected with paper-based questionnaires. Eye movements were recorded with the SMI iView X Hi-Speed system (sampling rate of $240 \mathrm{~Hz}$ ). The tracking appliance was placed in front of a 23 " monitor (resolution of 1920x1080, 96 PPI) showing the process model to the participants. For calibration, a 13-point calibration was performed. Eye tracking data collected during the study was analyzed and visualized with SMI BeGaze 3.7. Finally, SPSS 25 was used for all statistical analyses.

\section{DATA ANALYSIS AND INTERPRETATION}

In Table II, for each participant (i.e., ID), the number of fixations, average fixation duration (in ms), comprehension question score, intrinsic, extraneous, germane cognitive load, perceived usefulness for understandability, and perceived ease of understandability are shown. Note that for participants 1,4 , 


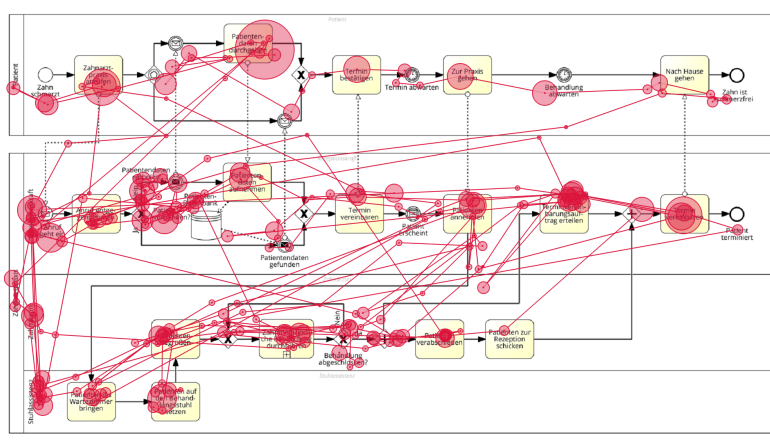

(a) Scan path (i.e., sequence of fixations) from a participant

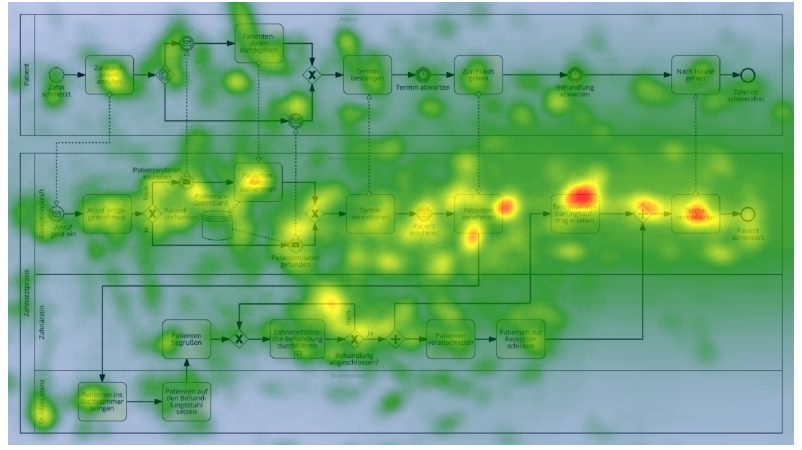

(b) Heat Map (i.e., density of fixations after model comprehension)

Fig. 2. Visualization of recorded eye movements

and 6, no eye tracking data are available because recording was not possible due to issues with the eye movement calibration (e.g., strabism). The mean and standard deviation of all participants in all performance measures are presented as well.

The participants fixated in average 652.53 points (e.g., modeling constructs) during model comprehension. The average fixation duration alluded a medium level of interaction with the model elements during comprehension (i.e., fixation duration typically ranges from 150 to $300 \mathrm{~ms}$ ). Participants achieved an average score of 5.87 ( $\max$. is 8 ). This indicates that the presented dental appointment process model could be comprehended intuitively. Concerning cognitive load, while intrinsic (i.e., 3.53) and extraneous (i.e., 2.93) load were at a moderate level, the result for germane cognitive load (i.e., 5.87) indicated an above-average level. Hence, the mental effort for comprehending the information in the model was supported by constructed mental schemata, which led to a more proper comprehension. Regarding level of acceptability, the results for PUU (i.e., 15.43) and PEU (i.e., 15.81) were on average (i.e., max. is 28 respectively), which indicated that the participants were indecisive regarding the benefits of presenting process information in a BPMN process model.

Regarding eye movements, scan paths (i.e., chronological alignment of fixation sequence) during model comprehension with related properties (e.g., fixation) were considered. Fig. 2 (a) depicts a scan path from a participant. The dots represent fixations (i.e., the larger the dot, the longer the dwell time) and the connecting lines constitute eye movements from one fixation to another. The participant has looked at each element in the process model and it is recognizable by the fixations, which places in the model have been looked at more often and longer. It is noticeable that the constructs decision, loop, and parallelism have been considered frequently compared to other elements. Fig. 2 (b) shows the superimposed heat map of all participants. A heat map provides no information about individual fixations, in lieu the magnitude of visual attention for all participants is revealed. The heat map shown in Fig 2 (b) confirmed similar eye movements of all participants, as the eye movements of the participant in Fig. 2 (a). ${ }^{1}$.

\footnotetext{
${ }^{1}$ Materials and results are available at: tinyurl.com/1uops $3 \times 0$
}

\section{A. Inferential Statistics}

To evaluate whether person-related characteristics (see Tab. I) have an influence on process model comprehension, the participants were grouped based on the same characteristics. For each person-related characteristic, a two-independent Mann-Whitney U test was performed for each performance measure (see Sect. II). For the characteristic Profession, a oneway analysis of variance (ANOVA) was performed. Finally, the significance value was set to $\mathrm{p}<.05$.

1) Gender: The results of 8 female participants were compared with 8 males. Regarding the performance measure Fixation and Fixation Duration, the results of 7 female participants were compared with 6 males.

$$
\begin{array}{llll}
\text { Score: } & \mathrm{U}=29.0, & \mathrm{z}=-.33 ; & \mathrm{p}=.739 \\
\text { ICL: } & \mathrm{U}=18.5, & \mathrm{z}=-1.45 ; & \mathrm{p}=.148 \\
\text { ECL: } & \mathrm{U}=27.0, & \mathrm{z}=-.53 ; & \mathrm{p}=.597 \\
\text { GCL: } & \mathrm{U}=31.0, & \mathrm{z}=-.11 ; & \mathrm{p}=.913 \\
\text { PUU: } & \mathrm{U}=31.5, & \mathrm{z}=-.05 ; & \mathrm{p}=.739 \\
\text { PEU: } & \mathrm{U}=15.0, & \mathrm{z}=-1.81 ; & \mathrm{p}=.071 \\
\text { Fixation: } & \mathrm{U}=6.0, & \mathrm{z}=-2.14 ; & \mathrm{p}=.032 \\
\text { Fix. Dur.: } & \mathrm{U}=17.0, & \mathrm{z}=-.57 ; & \mathrm{p}=.568
\end{array}
$$

The $\mathrm{U}$ test indicated that the number of fixations was greater for female (Median $(\mathrm{Mdn})=704.0)$ than for male participants $(\mathrm{Mdn}=566.5)$.

2) Age: The results of 6 participants with an age between 20 - 25 were compared with 10 with an age between $26-35$. Regarding the performance measure Fixation and Fixation Duration, the results of 6 participants with an age between 20 - 25 were compared with 7 with an age between $26-35$.

$\begin{array}{llll}\text { Score: } & \mathrm{U}=23.0, & \mathrm{z}=-.80 ; & \mathrm{p}=.423 \\ \text { ICL: } & \mathrm{U}=25.0, & \mathrm{z}=-.55 ; & \mathrm{p}=.580 \\ \text { ECL: } & \mathrm{U}=18.0, & \mathrm{z}=-1.31 ; & \mathrm{p}=.190 \\ \text { GCL: } & \mathrm{U}=19.0, & \mathrm{z}=-1.25 ; & \mathrm{p}=.213 \\ \text { PUU: } & \mathrm{U}=23.5, & \mathrm{z}=-.72 ; & \mathrm{p}=.470 \\ \text { PEU: } & \mathrm{U}=20.0, & \mathrm{z}=-1.10 ; & \mathrm{p}=.272 \\ \text { Fixation: } & \mathrm{U}=7.0, & \mathrm{z}=-2.00 ; & \mathrm{p}=.046 \\ \text { Fix. Dur.: } & \mathrm{U}=20.0, & \mathrm{z}=-.14 ; & \mathrm{P}=.886\end{array}$

The $\mathrm{U}$ test indicated that the number of fixations was greater for participants with an age between $20-25(\mathrm{Mdn}=713.5)$ than for an age between $26-35(\mathrm{Mdn}=605.0)$.

3) Profession: The results of 1 epidemiologist, 4 economists, 4 computer scientists, 4 medical practitioners, 2 
biologists, and 1 psychologist were compared with each other. Therefore, the performance measure Fixation and Fixation Duration, the results of 4 economists, 3 computer scientists, 3 medical practitioners, 2 biologists, and 1 psychologist were compared with each other.

$\begin{array}{llll}\text { Score: } & \mathrm{F}(5,10)= & 1.52 ; & \mathrm{p}=.268 \\ \text { ICL: } & \mathrm{F}(5,10)= & 2.51 ; & \mathrm{p}=.053 \\ \text { ECL: } & \mathrm{F}(5,10)= & 2.67 ; & \mathrm{p}=.088 \\ \text { GCL: } & \mathrm{F}(5,10)= & 1.52 ; & \mathrm{p}=.269 \\ \text { PUU: } & \mathrm{F}(5,10)= & 2.57 ; & \mathrm{p}=.096 \\ \text { PEU: } & \mathrm{F}(5,10)= & 1.65 ; & \mathrm{p}=.234 \\ \text { Fixation: } & \mathrm{F}(4,12)=.646 ; & \mathrm{p}=.645 \\ \text { Fix. Dur.: } & \mathrm{F}(4,12)=.233 ; & \mathrm{p}=.912\end{array}$

No significant differences were found.

4) Expertise: The results of 8 participants with process model expertise were compared with 8 without expertise. Regarding the performance measure Fixation and Fixation Duration, the results of 6 participants with process model expertise were compared with 7 without expertise.

$$
\begin{array}{llll}
\text { Score: } & \mathrm{U}=31.5, & \mathrm{z}=-.06 ; & \mathrm{p}=.956 \\
\text { ICL: } & \mathrm{U}=8.5, & \mathrm{z}=-2.52 ; & \mathrm{p}=.012 \\
\text { ECL: } & \mathrm{U}=8.5, & \mathrm{z}=-2.49 ; & \mathrm{p}=.013 \\
\text { GCL: } & \mathrm{U}=30.5, & \mathrm{z}=-.17 ; & \mathrm{p}=.869 \\
\text { PUU: } & \mathrm{U}=32.0, & \mathrm{z}=.00 ; & \mathrm{p}=1.00 \\
\text { PEU: } & \mathrm{U}=29.0, & \mathrm{z}=-.32 ; & \mathrm{p}=.747 \\
\text { Fixation: } & \mathrm{U}=8.0, & \mathrm{z}=-1.86 ; & \mathrm{p}=.063 \\
\text { Fix. Dur.: } & \mathrm{U}=14.0, & \mathrm{z}=-1.00 ; & \mathrm{p}=.317
\end{array}
$$

The $\mathrm{U}$ test indicated that the intrinsic cognitive load was greater for participants without process model expertise (Mdn $=4)$ than for participants with expertise $(\mathrm{Mdn}=3.25)$. The $U$ test indicated that the extrinsic cognitive load was greater for participants without process model expertise $(\mathrm{Mdn}=3)$ than for participants with expertise $(\mathrm{Mdn}=2)$.

5) Technical Affinity in Profession: The results of 8 participants without technical affinity in their profession were compared with 8 with technical affinity. Regarding the performance measure Fixation and Fixation Duration, the results of 7 participants without technical affinity in their profession were compared with 6 with a technical affinity.

$$
\begin{array}{llll}
\text { Score: } & \mathrm{U}=19.5, & \mathrm{z}=-1.39 ; & \mathrm{p}=.166 \\
\text { ICL: } & \mathrm{U}=25.5, & \mathrm{z}=-.69 ; & \mathrm{p}=.486 \\
\text { ECL: } & \mathrm{U}=31.5, & \mathrm{z}=-.05 ; & \mathrm{p}=.958 \\
\text { GCL: } & \mathrm{U}=18.0, & \mathrm{z}=-1.54 ; & \mathrm{p}=.125 \\
\text { PUU: } & \mathrm{U}=30.0, & \mathrm{z}=-.22 ; & \mathrm{p}=.830 \\
\text { PEU: } & \mathrm{U}=22.0, & \mathrm{z}=-1.06 ; & \mathrm{p}=.288 \\
\text { Fixation: } & \mathrm{U}=19.0, & \mathrm{z}=-.29 ; & \mathrm{p}=.775 \\
\text { Fix. Dur.: } & \mathrm{U}=12.0, & \mathrm{z}=-1.29 ; & \mathrm{p}=.199
\end{array}
$$

No significant differences were found.

6) Technical Affinity in Leisure: The results of 13 participants without a technical affinity in their leisure were compared with those of 3 with a technical affinity in their leisure. Regarding the performance measure Fixation and Fixation Duration, the results of 11 participants without a technical affinity in their leisure were compared with those of 2 with a technical affinity in their leisure.

$$
\begin{array}{llll}
\text { Score: } & \mathrm{U}=13.5, & \mathrm{z}=-.85 ; & \mathrm{p}=.394 \\
\text { ICL: } & \mathrm{U}=7.5, & \mathrm{z}=-1.65 ; & \mathrm{p}=.099 \\
\text { ECL: } & \mathrm{U}=14.5, & \mathrm{z}=-.68 ; & \mathrm{p}=.498 \\
\text { GCL: } & \mathrm{U}=16.0, & \mathrm{z}=-.49 ; & \mathrm{p}=.623 \\
\text { PUU: } & \mathrm{U}=18.0, & \mathrm{z}=-.21 ; & \mathrm{p}=.836 \\
\text { PEU: } & \mathrm{U}=16.5, & \mathrm{z}=-.41 ; & \mathrm{p}=.683 \\
\text { Fixation: } & \mathrm{U}=6.0, & \mathrm{z}=-.99 ; & \mathrm{p}=.324 \\
\text { Fix. Dur.: } & \mathrm{U}=9.0, & \mathrm{z}=-.40 ; & \mathrm{p}=.693
\end{array}
$$

No significant differences were found.

\section{B. Discussion and Next Steps}

The results revealed that the comprehension of the process model was intuitively possible for healthcare professionals. Although individual results were significant, no person-related characteristic has been identified, which considerably favors process model comprehension. Expertise in process modeling assisted the working memory load during model comprehension. The summarized results of all participants from the study indicated an average interactivity of the process model elements and the presented process model was positively perceived. Particularly interesting was the above-average germane cognitive load. It appears that participants were not strongly mentally engaged and, hence, were not confronted with severe difficulties in handling the information shown in the process model. Regarding the level of acceptability, the results were on an average level, which implies that the behavioral intention to apply process model is not clear. Additionally, participants were indecisive in their attitude regarding the benefits of process models as a lingua franca for communication and collaboration. Regarding recorded eye movements, the descriptives show that similar comprehension strategies were applied, whereas the model constructs decision, loop, and parallelism have been considered most often by the participants. However, several observations pertaining to the eye movements showed that participants of older age needed fewer fixations and achieved the same comprehension question score juxtaposed to participants of younger age. This result may indicate person-related characteristics, which are influencing the nature of comprehending process models. In this study, we identified the characteristics gender, age, and expertise as such influencing factors that needed to be investigated in further studies (see Section IV-C). Importantly, participants without expertise in process modeling were able to comprehend the syntactic of the respective constructs. An explanation would be that the consideration of the semantics supports the derivation of the syntactics. The insights obtained from this study unraveled that BPMN process models might be a suitable lingua franca to foster the comprehensible exchange of information as well as collaboration between IT and healthcare professionals in the course of digitization. In order to get a better understanding, more studies and results are required. Therefore, the exploratory insights from this study can be used as connecting factor. These include, for example, the comprehension of more complex process models documenting different scenarios. In addition, the application of a latent variable model might reveal specific person-related characteristics, which can have a significant positive and a negative influence on the comprehension of such models. 
Attention should be paid on measures that increase the level of acceptability regarding the proper utilization and the related benefits in applying process models. Based on the preliminary results, we are planning a follow-up study. We will consider additional person-related characteristics, eye movements, and the electrodermal activity that may provide novel insights.

\section{Limiting Factors}

First, the process model might not be representative. Process models document procedures of the real world, which are more complex. However, the used process model was kept simple intentionally. Research showed that process model comprehension becomes more error-prone with increasing number of modeling elements [29]. Consequently, complex process models make different demands regarding the cognitive load and the level of acceptability. Second, the scenario documented in respective process model represents another limitation. Only one scenario (i.e., dental appointment) was presented and, hence, more different scenarios in process models might have a diverse influence on model comprehension. Third, the inherent difficulty (e.g., question difficulty) of the study material may not be appropriate. In detail, the true-or-false comprehension questions might be too easy, since the participants reached an above-average score. Fourth, another limitation lies in the recruitment of participants. Regarding several personrelated characteristics (e.g., profession, age), an imbalance was present, which could have affected the statistics. Fifth, the sample sizes limit the statistical power and there might be significant differences, which we could not detect, but which might become apparent in larger sample sizes. Finally, sixth, current COVID-19 hygiene regulations (e.g., permanent wearing of a face mask) may have influenced the participants.

\section{SUMMARY}

In the context of digitization, this paper presented the results from an exploratory eye tracking study on process model comprehension. The objective of this study was to contribute additional insights for providing a lingua franca to emerge new synergies between IT and healthcare professionals in terms of communication as well as collaboration. Professionals from healthcare were asked to read and comprehend a BPMN 2.0 process model, whilst their eye movements as well as information regarding the cognitive load and level of acceptability were captured. The results revealed that the process model can be comprehended intuitively and that BPMN 2.0 might be a candidate for a lingua franca in this context. While results look promising, however, further studies are needed as the study at hand was confronted several limitations (e.g., one involving more participants with balanced distribution of person-related characteristics as well as more diverse process models). The results foster our conceptual framework for process model comprehension in healthcare that incorporates methods and theories from cognitive neuroscience and psychology [30].

\section{ACKNOWLEDGMENT}

The study was carried out in the context of the translational medicine graduate program of the University of Würzburg.

\section{REFERENCES}

[1] M. M. Gobble, "Digitalization, digitization, and innovation," ResearchTechnology Management, vol. 61, no. 4, pp. 56-59, 2018.

[2] M. H. van Velthoven, C. Cordon, and G. Challagalla, "Digitization of healthcare organizations: the digital health landscape and information theory," Int J Medical Informatics, vol. 124, pp. 49-57, 2019.

[3] H. Sadeghian, F. Zokaei, and S. H. Jazi, "Constrained kinematic control in minimally invasive robotic surgery subject to remote center of motion constraint," Journal of Int \& Robo Sys, vol. 95, no. 3, pp. 901-913, 2019.

[4] M. Wehde, "Healthcare 4.0," IEEE Engineering Management Review, vol. 47, no. 3, pp. 24-28, 2019.

[5] G. Adamo et al., "What is a process model composed of?" Soft and Sys Mod, pp. 1-29, 2021.

[6] D. C. Wynn and P. J. Clarkson, "Process models in design and development," Res in Eng Des, vol. 29, no. 2, pp. 161-202, 2018.

[7] K. Figl, "Comprehension of procedural visual business process models," Bus \& Inf Sys Eng, vol. 59, no. 1, pp. 41-67, 2017.

[8] M. Zimoch et al., "Cognitive insights into business process model comprehension: Preliminary results for experienced and inexperienced individuals," in BPMDS'17, 2017, pp. 137-152.

[9] — - "Eye tracking experiments on process model comprehension: Lessons learned," in BPMDS'17, 2017, pp. 153-168.

[10] O. Turetken et al., "The effect of modularity representation and presentation medium on the understandability of business process models in bpmn," in BPM'16. Springer, 2016, pp. 289-307.

[11] J. Claes et al., "The structured process modeling method (spmm) what is the best way for me to construct a process model?" Decision Support Systems, vol. 100, pp. 57-76, 2017.

[12] K. Figl and J. Recker, "Exploring Cognitive Style and Task-Specific Preferences for Process Representations," in Req Eng, 2014, pp. 63-85.

[13] O. Turetken and Others, "Cognitive Style and Business Process Model Understanding," in CAiSE'17, 2017, pp. 72-84.

[14] M. Razavian et al., "When cognitive biases lead to business process management issues," in BPM'16, 2016, pp. 147-156.

[15] M. Winter et al., "Towards the applicability of measuring the electrodermal activity in the context of process model comprehension: Feasibility study," Sensors, vol. 20, no. 16, p. 4561, 2020.

[16] R. Petrusel et al., "How visual cognition influences process model comprehension," Dec Supp Sys, vol. 96, pp. 1 - 16, 2017.

[17] W. Wang et al., "Effect of Linked Rules on Business Process Model Understanding," in BPM'17, 2017, pp. 200-215.

[18] R. B. Duarte et al., "A systematic literature review on the usage of eye-tracking in understanding process models," BPMJ, 2020.

[19] M. Tallon et al., "Comprehension of business process models: Insight into cognitive strategies via eye tracking," ESWA, pp. 145-158, 2019.

[20] M. Ramos-Merino et al., "A bpmn based notation for the representation of workflows in hospital protocols," $J$ of MS, vol. 42, pp. 1-10, 2018.

[21] P. Carayon et al., "Challenges and opportunities for improving patient safety through human factors and systems engineering," Health Affairs, vol. 37, no. 11, pp. 1862-1869, 2018.

[22] OMG, "Object Management Group Specification. Business Process Modeling \& Notation 2.0," 2019.

[23] I. Krajbich, C. Armel, and A. Rangel, "Visual fixations and the computation and comparison of value in simple choice," Nature Neuroscience, vol. 13, no. 10, pp. 1292-1298, 2010.

[24] J. M. Henderson, W. Choi, S. G. Luke, and J. Schmidt, "Neural correlates of individual differences in fixation duration during natural reading," Quart J of Experimental Psychology, vol. 71, no. 1, pp. 314-323, 2018.

[25] F. Paas et al., "Cognitive load theory and instructional design: Recent developments," Edu Psych, vol. 38, no. 1, pp. 1-4, 2003.

[26] M. Klepsch, F. Schmitz, and T. Seufert, "Development and validation of two instruments measuring intrinsic, extraneous, and germane cognitive load," Frontiers in Psychology, vol. 8, Nov. 2017.

[27] W. R. King and J. He, "A meta-analysis of the technology acceptance model," Inf \& Man, vol. 43, no. 6, pp. 740-755, Sept. 2006.

[28] O. Turetken et al., "Influential characteristics of enterprise information system user interfaces," J of Comp Inf Sys, vol. 59, pp. 243-255, 2019.

[29] J. Mendling et al., "Understanding the occurrence of errors in process models based on metrics," in CoopIS'07, 2007, pp. 113-130.

[30] M. Zimoch et al., "Towards a Conceptual Framework Fostering Process Comprehension in Healthcare," in CBMS'17, 2017, pp. 167-168. 